\title{
SDQ (Strengths and Difficulties Questionnaire): Identificação de Características Comportamentais de Crianças Leitoras ${ }^{1}$
}

\author{
Luciene Stivanin ${ }^{2}$ \\ Claudia Ines Scheuer \\ Francisco Baptista Assumpção Jr \\ Universidade de São Paulo
}

\begin{abstract}
RESUMO - O objetivo deste estudo foi verificar características comportamentais em crianças com desenvolvimento típico de leitura, utilizando o Strengths and Difficulties Questionnaire (SDQ). Este breve questionário é uma medida útil em psicopatologia aplicada a crianças e jovens de 4 a 16 anos. O SDQ é dividido em cinco sub-escalas: problemas no comportamento pró-social, hiperatividade, problemas emocionais, de conduta e de relacionamento. Participaram da pesquisa pais e professores de 74 crianças, que cursavam a $2^{\mathrm{a}}, 3^{\mathrm{a}}$ ou $4^{\mathrm{a}}$ séries em uma escola pública de São Paulo. As crianças não apresentavam alterações no desenvolvimento geral e na linguagem, e nem problemas escolares. Pais e professores responderam ao questionário. As respostas ao SDQ diferiram entre pais e professores, e de acordo com o sexo e a série escolar das crianças. Estas diferenças podem refletir mudanças comportamentais e de comunicação, conseqüência de situações vivenciadas no período do desenvolvimento no qual se encontram.
\end{abstract}

Palavras-chave: Strengths and Difficulties Questionnaire; desenvolvimento típico; crianças leitoras; características comportamentais.

\section{SDQ (Strengths and Difficulties Questionnaire): Identification of Children Readers Behavioral Characteristics}

\begin{abstract}
The purpose of this study was to verify behavioral characteristics of children with typical reading development by means of the Strengths and Difficulties Questionnaire (SDQ). This brief questionnaire is a useful measure in psychopathology applied to children and young people from 4 to 16 years. The SDQ is divided in five subscales: prosocial behavior problems, hyperactivity, and emotional, conduct and relationship problems. The participants were parents and teachers of 74 children, attending $2^{\text {nd, }} 3^{\text {rd }}$, or $4^{\text {th }}$ grades in a public school in the city of São Paulo. The children had no general development and language disorders, and scholars problems. Parents and teachers answered the questionnaires. The answers to the SDQ differed between parents and teachers, and regarding to children's gender and grade. These differences may reflect communication and behavioral changes resulting from experienced situations in children's present developmental stage.
\end{abstract}

Keywords: Strengths and Difficulties Questionnaire; typical development; children reader; behavioral characteristics.

A literatura aponta para uma possível associação entre alterações comportamentais e de linguagem escrita (Bennet, Brown, Boyle \& Racine, 2003; Carroll, Maughan, Goodman \& Meltzer 2005; Fletcher, Shaywitz \& Shaywitz, 1999; Hinshaw, 1992; Horwitz \& cols., 2003; Irwin, Carter \& Briggs-Cowan, 2002; Linares-Orama, 2005, Mayes, Calhoun, \& Crowell, 2000; Rescorla, 2000; Willcutt \& Pennington, 2000). De acordo com Ferraz (2002), os problemas de leitura e escrita são descritos desde 1887, passando, desde então, por várias definições e abrangendo diversos termos. Envolvendo essas alterações, a Associação Americana de Psiquiatria (1994), em seu Manual Diagnóstico e Estatístico de Transtornos Mentais (DSM-IV), apresenta o Transtorno de Aprendizagem como "funcionamento acadêmico substancialmente abaixo do esperado, tendo em vista a idade cronológica, medidas de inteligência e educação apropriadas à idade" (p.

1 Estudo derivado de parte da tese de Luciene Stivanin. Apoio financeiro da Fundação de Amparo à Pesquisa do Estado de São Paulo (FAPESP) - Processo 05/60488-0.

2 Endereço: Rua Teodoro Sampaio, 632, apto.52, Pinheiros. São Paulo, SP. CEP 05406-000.E-mail: stivanin@usp.br.
80), envolvendo os transtornos de leitura, da matemática e da aprendizagem sem outra especificação.

Outros quadros psiquiátricos, também descritos no DSM$I V$, podem apresentar uma associação com alterações de leitura e escrita, como o transtorno de déficit de atenção e hiperatividade (Fletcher \& cols., 1999; Mayes \& cols., 2000), o transtorno de conduta (Bennet \& cols., 2003; Hinsaw, 1992; Linares-Orama, 2005), e os transtornos de ansiedade e depressão (Willcutt \& Pennington, 2000).

Por outro lado, alterações na linguagem escrita podem contribuir para problemas comportamentais, emocionais e de relacionamento. Alguns autores relatam problemas como desatenção, mau comportamento em classe, desenvolvimento de quadros de ansiedade ou depressão decorrente dos repetidos episódios de fracasso escolar, dificuldade de adaptação e para superar as demandas sociais, expressão de fúria e raiva, desistência do processo de aprendizagem e utilização de estratégias para evitar esta situação (e.g., Carroll \& cols., 2005; Sisto, 2001; Sisto \& Fernandes, 2004).

De acordo com Rutter (1987), o desenvolvimento típico ocorre na interação de diversas áreas como linguagem, 
cognição, comportamento e emoção. Nesse sentido, pode ser observado o efeito do comportamento e das emoções na linguagem e aprendizagem: quando a criança é capaz de regular seu estado interno (manutenção do estado fisiológico, estabelecimento do sentimento de segurança, experiência de emoções positivas e controle das emoções negativas), ela pode manter o foco da atenção nos objetivos externos (utilização da linguagem, interação com os outros, exploração dos objetos), podendo assim aprender e se adaptar ao ambiente (Hay, Payne \& Chadwick, 2004; Tronick, 1989). Por outro lado, o desenvolvimento da linguagem também contribui para o estado emocional e comportamental. Com a capacidade para expressar opiniões, sentimentos, argumentar e entender crenças, idéias e desejos das outras pessoas, as crianças têm condições de criar soluções para resolver os problemas interpessoais, não necessitando criar conflitos e agressões físicas na tentativa de transmitir suas necessidades (McElwain \& Volling, 2002).

Embora haja uma relação entre problemas de linguagem (oral e escrita), quadros comportamentais e emocionais, crianças com desenvolvimento típico e sem queixas também apresentam riscos para problemas psiquiátricos, o que pode interferir nas relações interpessoais e aprendizagens posteriores.

Para rastrear características comportamentais, emocionais e sociais, existem instrumentos elaborados em forma de escalas e/ou questionários. Esses instrumentos, destinados ao rastreamento de problemas mentais, são, entre outros, auxiliares no diagnóstico e permitem melhor compreensão do desenvolvimento infantil. Questionários e escalas são largamente utilizados porque são de rápida e fácil aplicação, pontuação e interpretação, e podem ser respondidos pela criança, por pais, cuidadores e professores (Barbosa, Gouveia \& Barbosa, 2003).

Entre os instrumentos conhecidos e utilizados no Brasil, encontra-se o Questionário de Capacidades e Dificuldades (Strengths and Difficulties Questonnaire, SDQ), aplicado a pais e professores, construído em 1997 por Goodman e validado no Brasil em 2000 por Fleitlich, Cartázar e Goodman. O SDQ é um questionário que rastreia problemas de saúde mental infantil em cinco áreas: problemas no comportamento pró-social, hiperatividade, problemas emocionais, de conduta e de relacionamento. As vantagens na utilização do SDQ foram evidenciadas em relação à formatação mais compacta, maior focalização das capacidades e dificuldades, melhores informações sobre dificuldades de atenção/hiperatividade, relação com colegas e comportamento pró-social (Fleitlich \& cols., 2000).

Cury e Golfeto (2003), aplicando o SDQ a pais de crianças de $1^{\text {a }}$ a $4^{\text {a }}$ séries, obtiveram os seguintes scores: $16,8 \%$ de hiperatividade, $30,8 \%$ de sintomas emocionais, $17,7 \%$ de alterações de conduta e $14 \%$ de problemas nas relações interpessoais. As informações dadas por professores apontaram para: $8,25 \%$ de hiperatividade, $1,83 \%$ de sintomas emocionais, $8,25 \%$ de alterações de conduta e $2,75 \%$ de problemas nas relações interpessoais. Nesse estudo, porém, não houve discriminação entre escolares com e sem dificuldades na linguagem escrita.

Por esse motivo, o objetivo deste estudo foi conhecer, por meio de questionários, as características comportamentais em crianças com desenvolvimento típico de leitura, na perspectiva de pais e professores. Os questionários foram aplicados a pais e professores a fim de analisar o comportamento da criança em diferentes contextos.

\section{Método}

\section{Participantes}

Participaram deste estudo pais e professores de 74 crianças leitoras, de ambos os gêneros, cursando a $2^{\mathrm{a}}, 3^{\mathrm{a}}$ ou $4^{\mathrm{a}}$ séries do Ensino Fundamental da escola pública municipal Professor Olavo Pezzotti, localizada em São Paulo (SP). Na perspectiva de pais e professores, as crianças eram falantes e não apresentavam histórico de fracasso escolar, repetência, evasão, indicação de classe especial, déficits visuais não corrigidos, queixas de problemas auditivos e alterações no desenvolvimento.

A média de idade dos alunos de cada série foi: $2^{\mathrm{a}}$ série (8,6 anos), $3^{\mathrm{a}}$ série $\left(9,5\right.$ anos) e $4^{\mathrm{a}}$ série (10,7 anos). Em todas as séries havia aproximadamente $50 \%$ de crianças do gênero masculino e $50 \%$ do feminino.

Esta pesquisa foi aprovada pela Comissão de Ética para Análise de Projetos de Pesquisa do Hospital das Clínicas da Faculdade de Medicina da Universidade de São Paulo (protocolo de pesquisa ${ }^{\circ} 637 / 03$ ). Responsáveis pelas crianças assinaram o Termo de Consentimento Pós-Informação do Hospital das Clínicas da Faculdade de Medicina da Universidade de São Paulo.

\section{Material e procedimentos}

Para a seleção das crianças, cujos pais deveriam responder aos questionários, foram utilizados:

- Anamnese, elaborada pelos pesquisadores para a obtenção de dados sobre o desenvolvimento e a saúde da criança. Continha questões sobre as condições pré, peri e pós-natais, desenvolvimento neuropsicomotor e de linguagem, audição, visão, escolarização, comportamento e saúde (doenças infantis, medicamentos e tratamentos realizados);

- Questionário escolar, elaborado pela pesquisadora, para obter informações sobre o histórico escolar do aluno, eventuais dificuldades, reprovação, necessidade de reforço e problemas de comportamento como agitação, retraimento, agressividade etc.;

- Prova de leitura, criada exclusivamente para esta pesquisa. Essa prova consistiu na leitura em voz alta de 96 palavras, apresentadas isoladamente em um programa computacional. Foram excluídas as crianças que apresentaram dificuldades de leitura não esperadas para suas idades e séries escolares.

Para a obtenção de informações sobre características comportamentais e emocionais, foi aplicado o SDQ (Goodman, 1997; Fleitlich \& cols., 2000) aos pais e professores. Os pais responderam aos SQD em sessões individuais, cujas questões foram explicadas e aplicadas pela pesquisadora, em uma única sessão. Os professores também recebiam explicações sobre o preenchimento do questionário individualmente, mas só deveriam respondê-lo após 15 dias de observação do aluno na sala de aula.

O SDQ é um questionário que rastreia problemas de saúde mental infantil, aplicado aos pais e professores. É constitu- 
ído por 25 itens divididos em cinco sub-escalas: problemas no comportamento pró-social, hiperatividade, problemas emocionais, de conduta e de relacionamento, com cinco itens em cada sub-escala. As respostas podem ser: falso, mais ou menos verdadeiro ou verdadeiro, e cada item recebe uma pontuação específica. A soma de cada escala e a soma total permite a classificação da criança em três categorias: desenvolvimento normal (DN), limítrofe (DL) ou anormal (DA). Na sub-escala comportamento pró-social, quanto maior a pontuação, menor é a quantidade de queixas. Nas outras subescalas (hiperatividade, problemas emocionais, de conduta e de relacionamento), quanto maior a pontuação, maior o número de queixas.

\section{Resultados}

A Tabela 1 apresenta a porcentagem de crianças na categoria desenvolvimento anormal (DA) de acordo com a pontuação obtida no SDQ, nas cinco subescalas para pais e professores.
$\mathrm{Na}$ subescala comportamento pró-social, apenas uma criança obteve pontuação, tanto com pais quanto com professores, o que a inseriu na categoria DA. De forma geral, as subescalas com maior porcentagem de crianças na categoria DA foram as de problemas emocionais e de conduta (44\%), seguida por hiperatividade (40\%), na óptica dos pais. De forma geral, as respostas dos pais contribuíram para uma pontuação compatível com a categoria DA nas subescalas, mais do que as respostas dos professores. Respostas referentes a características de hiperatividade e problemas de conduta foram observadas principalmente nos meninos, enquanto os problemas emocionais foram mais exibidos pelas meninas.

$\mathrm{Na}$ Tabela 2 podem ser observados a média, o desviopadrão e o valor $p$ da comparação entre a pontuação obtida no SDQ para pais e a obtida no SDQ para professores. A média e o desvio-padrão em cada subescala do SDQ foram calculados e aplicados os testes t-pareado na comparação intragrupo e $o$ t-independente na comparação inter-grupo. Convém ressaltar que na subescala problemas no comportamento pró-social, uma pontuação alta indica menos

Tabela 1. Porcentagem de crianças na categoria "desenvolvimento anormal” (DA) de acordo com a pontuação no SDQ

\begin{tabular}{|c|c|c|c|c|c|c|c|c|}
\hline & & \multicolumn{2}{|c|}{$2^{a}$ série } & \multicolumn{2}{|c|}{$3^{a}$ série } & \multicolumn{2}{|c|}{$4^{a}$ série } & \multirow{2}{*}{$\begin{array}{l}\text { Total } \\
n=25\end{array}$} \\
\hline & & $F(n=13)$ & $M(n=12)$ & $F(n=12)$ & $M(n=12)$ & $F(n=14)$ & $M(n=11)$ & \\
\hline \multirow{2}{*}{$\begin{array}{l}\text { Problemas no comportamento } \\
\text { pró-social }\end{array}$} & Pais & $0,0(n=0)$ & $8,3(\mathrm{n}=1)$ & $0,0(\mathrm{n}=0)$ & $0,0(\mathrm{n}=0)$ & $0,0(\mathrm{n}=0)$ & $0,0(\mathrm{n}=0)$ & $4(n=1)$ \\
\hline & Prof & $0,0(n=0)$ & $8,3(n=1)$ & $0,0(\mathrm{n}=0)$ & $0,0(n=0)$ & $0,0(\mathrm{n}=0)$ & $0,0(\mathrm{n}=0)$ & $4(n=1)$ \\
\hline \multirow{2}{*}{ Hiperatividade } & Pais & $15,4(\mathrm{n}=2)$ & $16,7(n=2)$ & $16,7(n=2)$ & $8,3(\mathrm{n}=1)$ & $7,1(\mathrm{n}=1)$ & $18,1(n=2)$ & $40(n=10)$ \\
\hline & Prof & $7,7(n=1)$ & $16,6(n=2)$ & $0,0(\mathrm{n}=0)$ & $8,3(\mathrm{n}=1)$ & $0,0(\mathrm{n}=0)$ & $0,0(\mathrm{n}=0)$ & $16(n=4)$ \\
\hline \multirow{2}{*}{ Problemas emocionais } & Pais & $7,7(\mathrm{n}=1)$ & $16,7(n=2)$ & $16,7(n=2)$ & $16,7(\mathrm{n}=2)$ & $21,5(n=3)$ & $9,1(\mathrm{~N}=1)$ & $44(n=11)$ \\
\hline & Prof & $15,4(n=2)$ & $0,0(n=0)$ & $8,3(n=1)$ & $0,0(n=0)$ & $7,1(\mathrm{n}=1)$ & $0,0(n=0)$ & $16(n=4)$ \\
\hline \multirow{2}{*}{ Problemas de conduta } & Pais & $15,4(n=2)$ & $33,3(n=4)$ & $8,3(n=1)$ & $16,7(n=2)$ & $7,1(\mathrm{n}=1)$ & $9,1(\mathrm{~N}=1)$ & $44(n=11)$ \\
\hline & Prof & $7,7(n=1)$ & $25,0(n=3)$ & $0,0(\mathrm{n}=0)$ & $8,3(\mathrm{n}=1)$ & $0,0(\mathrm{n}=0)$ & $0,0(n=0)$ & $20(n=5)$ \\
\hline \multirow{2}{*}{ Problemas de relacionamento } & Pais & $7,7(n=1)$ & $8,3(\mathrm{n}=1)$ & $0,0(\mathrm{n}=0)$ & $0,0(\mathrm{n}=0)$ & $7,1(\mathrm{n}=1)$ & $18,1(n=2)$ & $20(n=5)$ \\
\hline & Prof & $7,7(n=1)$ & $8,3(\mathrm{n}=1)$ & $0,0(\mathrm{n}=0)$ & $16,7(\mathrm{n}=2)$ & $0,0(\mathrm{n}=0)$ & $0,0(\mathrm{n}=0)$ & $16(n=4)$ \\
\hline
\end{tabular}

Prof- professores; F-feminino; M-masculino

Tabela 2. Médias obtidas com os pais e professores em cada sub-escala do SQD.

\begin{tabular}{|c|c|c|c|c|c|c|}
\hline Série & Informante & $\begin{array}{c}\text { Problemas no } \\
\text { comportamento } \\
\text { pró-social } \\
\text { M (DP) }\end{array}$ & $\begin{array}{c}\text { Hiper } \\
\text { atividade } \\
\text { M (DP) }\end{array}$ & $\begin{array}{c}\text { Problemas } \\
\text { emocionais } \\
\text { M (DP) }\end{array}$ & $\begin{array}{l}\text { Problemas de } \\
\text { conduta } \\
\text { M (DP) }\end{array}$ & $\begin{array}{l}\text { Problemas de } \\
\text { relacionamento } \\
\text { M (DP) }\end{array}$ \\
\hline \multirow{3}{*}{$2^{\mathrm{a}}$} & Pais & $8,52(1,8)$ & $3,68(2,9)$ & $1,88(1,9)$ & $2,08(2,6)$ & $1,16(1,9)$ \\
\hline & Professores & $8,48(1,8)$ & $3,04(2,7)$ & $0,8(1,3)$ & $1,24(2,1)$ & $0,6(1,2)$ \\
\hline & $p$ & 0,873 & 0,191 & $0,022^{*}$ & 0,065 & $0,041^{*}$ \\
\hline \multirow{3}{*}{$3^{\mathrm{a}}$} & Pais & $8,67(1,6)$ & $3,42(2,6)$ & $1,70(16)$ & $1,5(1,7)$ & $0,958(0,9)$ \\
\hline & Professores & $9,92(1,4)$ & $1,292(1,8)$ & $0,83(1,4)$ & $0,625(1,13)$ & $0,708(1,19)$ \\
\hline & $p$ & 0,155 & $0,002^{*}$ & $0,007^{*}$ & $0,033^{*}$ & 0,388 \\
\hline \multirow{3}{*}{$4^{\mathrm{a}}$} & Pais & $8,44(1,7)$ & $2,76(2,6)$ & $1,76(2,1)$ & $1,2(1,7)$ & $1,16(1,5)$ \\
\hline & Professores & $8,56(1,5)$ & $1,92(1,8)$ & $1,84(2,0)$ & $0,44(0,8)$ & $0,64(0,9)$ \\
\hline & $p$ & 0,788 & 0,105 & 0,867 & $0,006^{*}$ & 0,091 \\
\hline
\end{tabular}

$\mathrm{M}=$ média; $\mathrm{DP}=$ desvio padrão

$\mathrm{p}<0,05$ 
problemas, enquanto nas outras subescalas, quanto maior a pontuação, maior o índice de queixas.

As características mais relatadas por pais e professores referem-se à hiperatividade, enquanto os problemas de relacionamento foram os menos referidos. Os pais relataram mais problemas do que os professores na maioria das subescalas. As diferenças estatisticamente significantes na pontuação entre pais e professores encontram-se nas subes- calas problemas emocionais e de relacionamento na $2^{\mathrm{a}}$ série, hiperatividade, problemas emocionais e de relacionamento na $3^{\mathrm{a}}$ série, e problemas de conduta na $4^{\mathrm{a}}$ série.

Na Tabela 3 estão as comparações da pontuação de cada sub-escala do SDQ levando em conta o gênero. Os resultados dessa tabela, referentes ao SDQ para pais, apontam que embora não fosse observada diferença estatisticamente significante entre os gêneros, as crianças do gênero masculino apresentaram

Tabela 3. Comparação segundo o gênero para cada sub-escala do SDQ para pais e professores.

\begin{tabular}{ccccccc}
\hline Informante & Gênero & $\begin{array}{c}\text { Problemas no } \\
\text { comportamento } \\
\text { pró-social } \\
\mathbf{M} \text { (DP) }\end{array}$ & $\begin{array}{c}\text { Hiperatividade } \\
\text { M (DP) }\end{array}$ & $\begin{array}{c}\text { Problemas } \\
\text { emocionais } \\
\text { M (DP) }\end{array}$ & $\begin{array}{c}\text { Problemas de } \\
\text { conduta } \\
\text { M (DP) }\end{array}$ & $\begin{array}{c}\text { Problemas de } \\
\text { relacionamento } \\
\text { M (DP) }\end{array}$ \\
\hline \multirow{2}{*}{ Pais } & Masculino & $8,17(1,9)$ & $3,46(2,75)$ & $1,6(1,68)$ & $1,83(2,01)$ & $1,11(1,66)$ \\
& Feminino & $8,87(1,51)$ & $2,9(2,7)$ & $1,95(2,1)$ & $1,38(2,2)$ & $1,08(1,4)$ \\
& $p$ & 0,086 & 0,384 & 0,433 & 0,367 & 0,917 \\
\hline \multirow{2}{*}{ Professores } & Masculino & $8,49(1,67)$ & $2,6(2,53)$ & $0,71(1,13)$ & $1,14(1,68)$ & $0,71(1,05)$ \\
& Feminino & $9,03(1,6)$ & $1,64(1,99)$ & $1,56(2,0)$ & $0,44(1,25)$ & $0,59(1,23)$ \\
& $p$ & 0,161 & $0,047 *$ & $0,028^{*}$ & $0,047 *$ & 0,639 \\
\hline
\end{tabular}

$\mathrm{M}=$ média; $\mathrm{DP}=$ desvio padrão $\mathrm{p}<0,05$

Tabela 4. Comparação entre séries para cada sub-escala do SDQ para PAIS.

\begin{tabular}{ccccccc}
\hline & & $\begin{array}{c}\text { Problemas no } \\
\text { comportamento } \\
\text { pró-social }\end{array}$ & $\begin{array}{c}\text { Hiper } \\
\text { atividade }\end{array}$ & $\begin{array}{c}\text { Problemas } \\
\text { emocionais }\end{array}$ & $\begin{array}{c}\text { Problemas de } \\
\text { conduta }\end{array}$ & $\begin{array}{c}\text { Problemas de } \\
\text { relacionamento }\end{array}$ \\
\hline \multirow{2}{*}{$2^{\text {a }}$ série } & $\mathrm{M}$ & 8,52 & 3,68 & 1,88 & 2,08 & 1,16 \\
& $\mathrm{DP}$ & 1,851 & 2,926 & 1,986 & 2,644 & 1,93 \\
\hline \multirow{2}{*}{$3^{\text {a }}$ série } & $\mathrm{M}$ & 8,667 & 3,042 & 1,708 & 1,794 & 0,958 \\
& $\mathrm{DP}$ & 1,659 & 2,645 & 1,654 & 1,2 & 1,999 \\
\hline \multirow{2}{*}{$4^{\mathrm{a}}$ série } & $\mathrm{M}$ & 8,44 & 2,76 & 1,76 & 1,732 & 1,519 \\
\hline & $\mathrm{DP}$ & 1,734 & 2,666 & 2,146 & 0,328 & 0,870 \\
\hline
\end{tabular}

$\mathrm{M}=$ média; $\mathrm{DP}=$ desvio padrão

$\mathrm{p}<0,05$

Tabela 5. Comparação entre séries para cada sub-escala do SDQ para PROFESSORES.

\begin{tabular}{|c|c|c|c|c|c|c|}
\hline & & $\begin{array}{c}\text { Problemas no } \\
\text { comportamento } \\
\text { pró-social }\end{array}$ & $\begin{array}{c}\text { Hiper } \\
\text { atividade }\end{array}$ & $\begin{array}{l}\text { Problemas } \\
\text { emocionais }\end{array}$ & $\begin{array}{l}\text { Problemas de } \\
\text { conduta }\end{array}$ & $\begin{array}{l}\text { Problemas de } \\
\text { relacionamento }\end{array}$ \\
\hline \multirow{2}{*}{$2^{a}$ série } & $\mathrm{M}$ & 8,48 & 3,04 & 0,8 & 1,24 & 0,6 \\
\hline & DP & 1,873 & 2,791 & 1,354 & 2,127 & 1,291 \\
\hline \multirow{2}{*}{$3^{\mathrm{a}}$ série } & $\mathrm{M}$ & 9,292 & 1,292 & 0,833 & 0,625 & 0,708 \\
\hline & DP & 1,429 & 1,829 & 1,435 & 1,135 & 1,197 \\
\hline \multirow{3}{*}{$4^{\mathrm{a}}$ série } & $\mathrm{M}$ & 8,56 & 1,92 & 1,84 & 0,44 & 0,64 \\
\hline & DP & 1,53 & 1,869 & 2,095 & 0,87 & 0,952 \\
\hline & $p$ & 0,166 & $\begin{array}{l}0,024^{*} \\
2^{\mathrm{a}}>3^{\mathrm{a}}=4^{\mathrm{a}}\end{array}$ & $\begin{array}{l}0,050^{*} \\
2^{\mathrm{a}}=3^{\mathrm{a}}<4^{\mathrm{a}}\end{array}$ & 0,145 & 0,947 \\
\hline
\end{tabular}

$\mathrm{M}=$ média; $\mathrm{DP}=$ desvio padrão $\mathrm{p}<0,05$ 
mais características em quase todas as subescalas, com exceção da subescala problemas emocionais, apresentados mais pelas meninas. Na óptica dos professores, os meninos apresentaram mais características de hiperatividade e problemas de conduta e as meninas, mais problemas emocionais.

As tabelas 4 e 5 contêm os dados da comparação intersérie, de acordo com pais e professores, respectivamente.

$\mathrm{Na}$ Tabela 4, embora não sejam observadas diferenças estatisticamente significativas entre as séries, a média da pontuação obtida na $2^{\text {a }}$ série foi maior, indicando mais queixas relatadas por pais de crianças desta série do que nas séries mais avançadas.

De acordo com os dados observados na Tabela 5 , na $2^{\text {a }}$ série houve mais alunos identificados com características de hiperatividade, pelos professores, do que na $3^{\mathrm{a}}$ e $4^{\mathrm{a}}$ séries. Por outro lado, na $4^{\text {a }}$ série, observou-se mais crianças com problemas emocionais do que na $2^{\mathrm{a}}$ e $3^{\mathrm{a}}$ séries.

\section{Discussão}

Os sintomas de problemas comportamentais são mais evidentes e preocupantes em crianças com problemas na leitura, associação largamente relatada na literatura (Bennet \& cols., 2003; Carroll \& cols., 2005; Fletcher \& cols., 1999; Hinshaw, 1992; Horwitz \& cols., 2003; Irwin \& cols., 2002; Linares-Orama, 2005; Mayes \& cols., 2000; Rescorla, 2000; Willcutt \& Pennington, 2000). Por isso, houve a preocupação em discriminar crianças com e sem problemas de desenvolvimento na leitura. O objetivo do presente trabalho foi estudar apenas as crianças com desenvolvimento típico de leitura e será discutida a comparação entre pais e professores, entre os gêneros e entre as séries escolares.

No que diz respeito à comparação entre pais e professores, os resultados deste estudo indicaram que as respostas dos pais preencheram mais critérios para a categoria "desenvolvimento anormal" do que as respostas dos professores, em todas as subescalas do SDQ. A falta de concordância entre vários informantes sobre a presença dos mesmos comportamentos em um mesmo indivíduo também é encontrada em outros trabalhos (Cury \& Golfeto, 2003; Fleitlich \& cols., 2000; Goodman, 1997). Uma criança pode apresentar diferentes comportamentos, de acordo com o lugar em que se encontra, com quem se relaciona e como é capaz de atender às diferentes demandas do ambiente multivariado. $\mathrm{O}$ professor tem dados de observação da criança na sala de aula, enquanto que os pais podem notar os comportamentos em casa, em lugares públicos e em outros contextos. Na sala de aula, a criança é exposta a regras impostas pela escola, lugar onde os comportamentos são mais facilmente controlados.

Porém, ao se considerar os sinais de hiperatividade, os mais relatados pelos participantes deste estudo, surge uma questão: as regras impostas pelos professores em sala de aula são capazes de controlar uma criança hiperativa? Essa questão leva à hipótese de que os sinais apontados por pais (em maior quantidade do que pelos professores) envolvem outros aspectos que não apenas os de hiperatividade, como a ausência e/ou dificuldade de imposição de limites durante o desenvolvimento.

Com relação à diferença entre gêneros, observa-se, na óptica de pais e de professores, que as crianças do gênero masculino tendem a apresentar mais hiperatividade, problemas de conduta e de relacionamento, enquanto as meninas apresentam mais problemas emocionais, diferença apontada de forma significante pelos professores.

A maior tendência das meninas para introjetar as dificuldades e somatizá-las pode relacionar-se à influência do meio cultural que as permite exibir mais queixas do que aos meninos. Os pais podem ainda manter algumas regras diferenciadas para educar meninos e meninas: os meninos são criados para serem fortes e desaconselha-se que demonstrem seus sentimentos, enquanto às meninas permitem-se mais comportamentos emotivos e expressão de sentimentos (Saud \& Tonelotto, 2005).

Por outro lado, a presença de hiperatividade e de problemas de conduta, predominantes em meninos, é apontada na literatura como uma característica masculina, interferindo, possivelmente, na relação interpessoal (Hay \& cols., 2004).

Ao comparar as características apresentadas entre as crianças de $2^{\mathrm{a}}, 3^{\mathrm{a}}$ e $4^{\mathrm{a}}$ séries, observou-se que a média da pontuação do SDQ tende a diminuir com a escolarização na maioria das subescalas do SDQ, o que também foi observado no estudo de Cury e Golfeto (2003). Maiores diferenças entre as séries foram observadas nas respostas dos professores, segundo os quais a hiperatividade está mais presente na $2^{\mathrm{a}}$ série (principalmente no gênero masculino), enquanto os problemas emocionais são mais evidentes na $4^{\text {a }}$ série (principalmente no gênero feminino).

Essa diferença de sinais observados entre as séries indica que as crianças (neste estudo, na faixa etária de 8,6 a 11 anos) podem criar novas estratégias em diversas situações e se expressar diferentemente com o desenvolvimento. As crianças mais novas da $2^{\mathrm{a}}$ série manifestam-se por atividade motora, ou por falta de recursos comunicativos mais eficientes e/ou ainda por não conseguirem nesta idade permanecer sentados por longo período de tempo. Já na $4^{\mathrm{a}}$ série, as crianças podem mais facilmente controlar seus atos motores e a compreensão mais desenvolvida das situações pode gerar expressões mais emocionais.

A diferença observada entre as variáveis estudadas (informantes, gênero e escolaridade) foi explicada por meio de argumentos teóricos encontrados na literatura. Todavia, um dado importante e que merece atenção é que a porcentagem de crianças que obtiveram pontuação para serem classificadas na categoria "desenvolvimento anormal" é maior do que a prevalência encontrada na população geral. No presente estudo, $40 \%$ das crianças foram consideradas DA de acordo com a pontuação na subescala hiperatividade, enquanto os estudos epidemiológicos apontam a prevalência de 3 a $6 \%$ de crianças com Transtorno do Déficit de Atenção e Hiperatividade (Rohde, Busnello \& Chachamovich, 1998). O mesmo foi observado para problemas emocionais - 44\% nesta pesquisa contra 10 a $15 \%$ em estudos epidemiológicos (DSM-IV).

De acordo com o DSM-IV, um quadro psicopatológico é caracterizado pela presença de perturbação em pelo menos dois ambientes. Neste estudo, o SDQ, instrumento que objetiva rastrear sinais psicopatológicos, foi aplicado a uma população de crianças com desenvolvimento típico, sem queixas por parte de pais e professores, a partir da observação 
em dois ambientes da criança: casa e escola. Assim, a porcentagem de crianças na categoria DA superior à prevalência encontrada na literatura, a diferença na caracterização do comportamento entre pais e professores e, principalmente entre as séries, fornecem alguns pontos importantes e que merecem atenção.

Os diferentes sinais apresentados ao longo da escolarização podem refletir as conseqüências de situações vivenciadas pelas crianças (neste caso, exclui-se dificuldades na linguagem escrita), mais do que sinais de um quadro patológico que vai se modificando. Em outras palavras, os comportamentos apresentados podem caracterizar diferentes formas de expressão, que se alteram com as possibilidades e as estratégias adquiridas durante o desenvolvimento, mais do que sinais da instalação de uma patologia.

Esse instrumento, com possibilidades de aplicação a vários acompanhantes da criança em desenvolvimento, permite uma coleta rápida e prática de dados sobre os comportamentos, em diferentes situações e com diversos interlocutores. Entretanto, deve-se ter cuidado e atenção à interpretação dos dados fornecidos por pais e professores, pois esses podem e parecem ter diferentes olhares, conhecimento e formas de falar ou expressar a respeito dos sujeitos por eles avaliados. Tais características deveriam ser associadas e correlacionadas com outras, tais como lugares que a criança freqüenta com os pais, entendimento dos pais e professores sobre os comportamentos, situações que os professores privilegiam na hora da avaliação, tipo de conduta escolar, dentre outros.

O objetivo do SDQ é rastrear problemas de saúde mental em cinco áreas. É um instrumento prático e rápido, permite a obtenção de dados oriundos de diferentes locais e interlocutores, mas deve ser cuidadosamente analisado, em vista dos pontos já destacados. Os sinais analisados devem servir não apenas como meio de prevenção. Uma vez que tais sinais caracterizam as estratégias utilizadas pelas crianças durante o desenvolvimento, podem ser vistos e utilizados para a criação de meios e ferramentas para o aprimoramento de outras habilidades, como a relação interpessoal, decisões, motivação, dentre outras.

Finalizando, os dados coletados pelo SDQ nem sempre são vistos como indícios de patologia e necessitam da complementação com outras informações. Devem ser utilizados como meio de prevenção e principalmente como estratégias na elaboração de condutas com a criança.

\section{Considerações Finais}

Os dados colhidos por meio do SDQ apontaram para sinais comportamentais relatados mais por pais do que por professores, para a existência de diferenças entre os gêneros e para a mudança de comportamentos entre as séries. O instrumento permitiu caracterizar os comportamentos em diferentes situações e com diferentes olhares, levando a necessidade de atenção e cuidado na interpretação dos dados. Os dados obtidos devem ser utilizados como medidas preventivas e de caracterização da criança em determinado momento do desenvolvimento e situação.

\section{Referências}

Associação Psiquiátrica Americana (1994). Manual diagnóstico e estatístico de distúrbios mentais. DSM-IV. São Paulo: Manole.

Barbosa, G. A., Gouveia, V. V., \& Barbosa, A. G. (2003). Escalas de avaliação em psiquiatria da infância e da adolescência. Em F. B. Assumpção Jr. \& E. Kuczynski (Orgs.), Tratado de psiquiatria da infância e da adolescência (pp. 121-129). São Paulo: Atheneu.

Bennet, K. J., Brown, S., Boyle, M. \& Racine, Y., \& Offord, D. (2003). Does low reading achievement at school entry cause conduct problems? Social Science and Medicine, 56, 24432448.

Carrol, J. M., Maughan, B., Goodman, R. \& Meltzer, H. (2005). Literacy difficulties and psychiatric disorders: Evidence for comorbidity. Journal of Child Psychology and Psychiatry, 46, 524-532.

Cury, C. R. \& Golfeto, J. H. (2003). Strengths and Difficulties Questionnaire (SDQ): A study of children in Ribeirão Preto. Revista Brasileira de Psiquiatria, 25(3), 139-145.

Ferraz, P. G. (2002). Transtornos da aprendizagem: proposta de um sistema tutorial para auxílio ao diagnóstico. Tese de Doutorado, Universidade de São Paulo, São Paulo.

Fleitlich, B., Cortazar, P. G. \& Goodman, R. (2000). Questionário de Capacidades e Dificuldades (SDQ). Revista Infanto (de Neuropsiquiatria da Infância e da Adolescência), 8, 44-50.

Fletcher, J. M., Schaywitz, S. E. \& Schaywitz, B.A. (1999). Comorbidity of learning and attention disorders. Pediatric Clinics of North America, 46, 885-897.

Goodman, R. (1997). The Strengths and Difficulties Questionnaire: A research note. Journal of Child Psychology and Psychiatry, 38, 581-586.

Hay, D. F., Payne, A. \& Chadwick, A. (2004). Peer relations in childhood. Journal of Psychology and Psychiatry, 45, 84-108.

Hinshaw, S. P. (1992). Externalizing behavior problems and academic underachievement in childhood and adolescence: Causal relationships and underlying mechanisms. Psychological Bulletin, 111, 127-155.

Horwitz, S. H., Irwin, J. R., Brigss-Cowan, M. J., Heenan, J. M. B., Mendoza, J. \& Carter, A. S. (2003). Language delay in a community cohort of young children. Journal of the American Academy of Child and Adolescent Psychiatry, 42, 932-940.

Irwin, J. R., Carter, A. S. \& Briggs-Cowan, M. J. (2002). The socialemotional development of "late-talking" toddlers. Journal of American Academy of Child and Adolescent Psychiatry, 41, 1324-1332.

Linares-Orama, N. (2005). Language-learning disorders and youth incarceration. Journal of Communication Disorders, 38, 311-319.

Mayes, S. D., Calhoun, S. I. \& Crowell, E. W. (2000). Learning disabilities and ADHD: Overlapping spectrum disorders. Journal of Learning Disabilities, 33, 417-424.

McElwain, N. L. \& Volling, B. (2002). Relating individual control, social understanding, and gender to child-friend interaction: A relationships perspective. Social Development, 11, 362-385.

Rescorla, L. (2000). Do late-talking toddlers turn out to have reading difficulties a decade later? Annals of Dyslexia, 50, 87-102. 
Rohde, L. A., Busnello, E. D. \& Chachamovich, E. (1998). Transtorno de déficit de atenção e hiperatividade: revisando conhecimentos. Revista ABP-APAL, 20, 166-178.

Rutter, M. (1987). The role of cognition in child development. British Journal of Medical Psychology, 60, 1-16.

Saud, L. F. \& Tonelotto, J. M. F. (2005). Comportamento social na escola: diferenças entre gênero e séries. Psicologia Escolar e Educacional, 9, 47-56.

Sisto, F. F. (2001). Dificuldades de aprendizagem em escrita: um instrumento de avaliação (Adape). Em F. F. Sisto, E. Boruchovitch, L. D. T. Fini, R. P. \& S. C. Brenelli Martinelli (Orgs.), Dificuldades de aprendizagem no contexto psicopedagógico (pp. 190-213). Petrópolis: Vozes.

Sisto, F. F. \& Fernandes, D. C. (2004). Dificuldades lingüísticas na aquisição da escrita e agressividade. Psicologia Escolar e Educacional, 8, 75-84.
Tronick, E. (1989). Emotions and emotional communication in infants. American Psychologist, 44, 112-119.

Willcutt, E. G. \& Pennington, B. F. (2000). Psychiatric comorbidity in children and adolescents with reading disability. Journal of Child Psychology and Psychiatry, 41, 1039-1048.

Recebido em 13.08.2007

Primeira decisão editorial em 02.01.2008

Versão final em 21.08.2008

Aceito em 05.03.2008 